Orthopäde

1997·26:749 @ Springer-Verlag 1997
Editorial

\section{Spondylolyse und Spondylolisthesis}

Dezeichnenderweise kommt die Erstbeschreibung der lumbalen Spondylolisthesis aus geburtshilflicher Feder. Neugebauer hat 1891 den Gleitwirbel als $\mathrm{Ge}$ burtshindernis beschrieben.

Seit damals sind isthmische und dysplastische Spondylolisthesen mit einer Fülle ungelöster Fragen verknüpft. Ätiologie und Pathogenese werden genauso kontrovers diskutiert wie die Indikation zu konservativer oder operativer Therapie, ganz zu schweigen von operativen Strategien der Stabilisierung mit und ohne Reposition.

In diesem Heft werden allein Fragen isthmischer Spondylolisthesis besprochen, die übrigen Formen des Wirbelgleitens finden hier keine Berücksichtigung.

Nach wie vor ist die Entstehung des Isthmusdefektes nicht endgültig geklärt. Während bisher überwiegend präexistente Dysplasien des Wirbelbogens, wie das Brocher vertreten hat, angenommen wurden, deuten Untersuchungen von Niethard darauf hin, daß diese Dysplasie und die Unterbrechung der Interartikularportion ebenso eine Folge gestörten Wirbelsäulenwachstums sind, wie die Spina bifida occulta, die in etwa $50 \%$ der Spondylolisthesen beobachtet wird. Gleichzeitig ergibt sich aus der Beachtung der Spina bifida auch ein wertvoller prognostischer Hinweis: Die Kombination von Spondylolyse und Spina bifida findet sich wesentlich häufiger bei schmerzhaften als bei symptomfreien Spondylolysen. Insgesamt wird der mechanische Aspekt der Lyseentstehung durch diese Untersuchungen unterstrichen. Die bekannte Häufung von Spondylolysen bei bestimmten mit Lordosebelastung einhergehenden Sportarten, die von Engelhardt dargestellt werden, unterstreichen diese Vorstellung. Die Beurteilung der sportlichen Belastbarkeit muß unter dem Aspekt der erfaßbaren Instabilität gesehen werden. Bei progredienten Gleitprozessen sollten Sportarten mit vermehrter axialer Belastung sowie Hyperextension und Rotation vermieden werden. Das kann bei der Frage nach der Schulsportbefreiung berücksichtigt werden. Stabile Spondylolysen und Olisthesen stellen natürlich keine Einschränkung für den Schulsport dar. Bei Kindern mit Rückenschmerzen beim Leistungssport muß sorgfältig nach Spondylolysen gefahndet werden, um schädigende Einflüsse ausschalten zu können.

In der Beantwortung der wichtigen Frage der Indikation zu konservativer oder operativer Behandlung im Kindesalter stellt D. Schlenzka ein praktikables Konzept vor, das bei prinzipiell konservativer Haltung allerdings bei höheren Gleitgraden von über $50 \%$ eine Stabilisierungsoperation immer vorsieht, $d a$ eine Progredienz des Gleitvorganges sicher angenommen werden muß. Er nimmt ebenso wie Hefti im nachfolgenden Beitrag zum „direct repair“der Fusion der Lysezone mit verschiedenen Osteosynthesetechniken Stellung. Wenn auch die Ausheilungsergebnisse von Morscher und Hefti unterhalb des 20. Lebensjahres über $95 \%$ betragen, muß doch die Diskusdegeneration des Gleitsegments in der Zukunft mit etwas
Sorge betrachtet werden, zumal echte Langzeitergebnisse noch fehlen.

Eine besondere Bedeutung kommt der Veränderung des Wirbelsäulenprofils durch das Wirbelgleiten zu. Jeder kennt die oft groteske Haltung des Spondyloptotikers und die charakteristische Veränderung seines Gangbildes. In geringerem Maße trifft das für alle hochgradigen kyphosierenden Spondylolisthesen zu. Dick hält deshalb bei einem lumbalen Kyphosewinkel von $85^{\circ}$ und einem Sacrumneigungswinkel von $35^{\circ}$ eine Kyphoseaufrichtung für wichtiger, als die Beseitigung der reinen Ventralverschiebung. Dieses Konzept wird an Ergebnissen über 9 Jahre überprüft.

Die 3 letzten Beiträge dieses Heftes aus Gießen, Ulm und Bad Wildungen befassen sich mit unterschiedlichem Schwerpunkt mit der Differential-Indikation zu Fusion in situ oder Repositionsspondylodese. Zur Vermeidung der bekannten neuralen Komplikationen werden unterschiedliche Techniken und Instrumentationen angegeben. Es hat den Anschein, daß durch leistungsfähigere Instrumentationen Risiken deutlich verringert und damit Indikationen $z u$ Repositionen häufiger gestellt werden können.

Alle Autoren bestätigen die engen Indikationsgrenzen der operativen Spondylolisthesis-Behandlung und betonten „unisono"das ärztliche Grundprinzip: Primum nil nocere.

\section{Hohmann \\ Die Herausgeber}

